Supporting Information

\title{
Isomerization Dynamics in the Symmetric and Asymmetric Fragmentation of Ethane Dications
}

Long Wei, ${ }^{\dagger}$ Chow-Shing Lam, ${ }^{\ddagger}$ Yu Zhang, ${ }^{*}$ " Baihui Ren, ${ }^{\dagger}$ Jie Han, ${ }^{\dagger}$ Bo Wang, ${ }^{\dagger}$ Yaming Zou, ${ }^{\dagger}$ Li Chen, ${ }^{\dagger}$ Kai-Chung Lau, ${ }^{*}+$ and Baoren Wei ${ }^{* \dagger}$

Institute of Modern Physics, Key Laboratory of Nuclear Physics and Ion-Beam Application (MOE), Fudan University, Shanghai 200433,

China

Department of Chemistry, City University of Hong Kong, Tat Chee Avenue, Kowloon Tong, Hong Kong, China

${ }^{\natural}$ College of Data Science, Jiaxing University, Jiaxing 314001, China

*E-mail: zyclay@,outlook.com

*E-mail: kaichung@,cityu.edu.hk

*E-mail: brwei@,fudan.edu.cn 


\section{The coincidence TOF map caused by $\mathrm{Ar}^{8+}$ ion collisions}

The coincidence TOF map of two ion-pairs $\mathrm{CH}_{3}{ }^{+}+\mathrm{CH}_{3}{ }^{+}$and $\mathrm{CH}_{2}{ }^{+}+\mathrm{CH}_{4}{ }^{+}$ produced by 3-keV/u $\mathrm{Ar}^{8+}$ ion impact on $\mathrm{C}_{2} \mathrm{H}_{6}$ is shown in Figure $\mathrm{S} 1$. The data within the black rectangle in Figures 1 and $\mathrm{S} 1$ is almost entirely (more than $\sim 97 \%$ ) contributed by the coincidence events $\mathrm{CH}_{3}{ }^{+}+\mathrm{CH}_{3}{ }^{+}$, and the events within the red rectangle are attributed only to the coincidence events $\mathrm{CH}_{2}{ }^{+}+\mathrm{CH}_{4}{ }^{+}$.

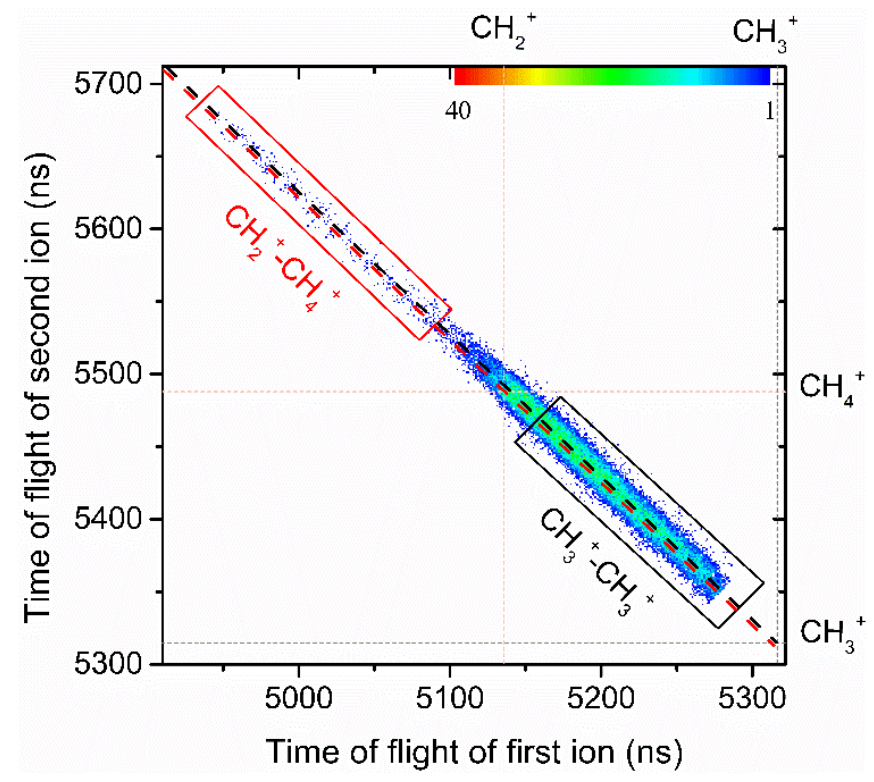

Figure $\mathbf{S 1}$ The coincidence TOF map of two-body fragmentation channels $\mathrm{CH}_{3}{ }^{+}+\mathrm{CH}_{3}{ }^{+}$and $\mathrm{CH}_{2}{ }^{+}$ $+\mathrm{CH}_{4}{ }^{+}$induced by $\mathrm{Ar}^{8+}$ ion impact.

\section{The 1D TOF spectra of the targeted dissociation channels}

The TOFs of the first and second recoil ions of the concerning fragment pairs, projected from corresponding coincidence maps in two collisions, are shown in Figure S2. A clear distribution difference can be seen for the two fragmentation channels. In Figure S2(a), the 1D TOF spectrum of $\mathrm{CH}_{2}{ }^{+}$fragment should be symmetrically distributed around the center of $\sim 4600$ ns (the TOF value of ions with initial kinetic energy of zero). The plateau in the lower TOF range is attributed to the $\mathrm{CH}_{2}^{+}$fragment. Thus the whole TOF range of $\mathrm{CH}_{2}{ }^{+}$is about $4450-4750$ ns. A similar plateau is also observed for the counterpart of $\mathrm{CH}_{2}{ }^{+}$, i.e., $\mathrm{CH}_{4}{ }^{+}$[see Figure S2(b)]. Subtracting the whole plateau of $\mathrm{CH}_{2}{ }^{+}$or $\mathrm{CH}_{4}{ }^{+}$, the residual peak of high intensity corresponds to the 
TOF of $\mathrm{CH}_{3}{ }^{+}$. As the channel $\mathrm{CH}_{3}{ }^{+}+\mathrm{CH}_{3}{ }^{+}$containing two identical fragments, the TOF of $\mathrm{CH}_{3}{ }^{+}$will distribute only on one side of the TOF center value ( $\left.\sim 760 \mathrm{~ns}\right)$. The deviation of $\mathrm{CH}_{3}{ }^{+}$TOF distribution from its center value is caused by the deadtime of the position sensitive detector, i.e., $\sim 35 \mathrm{~ns}$.

(a)

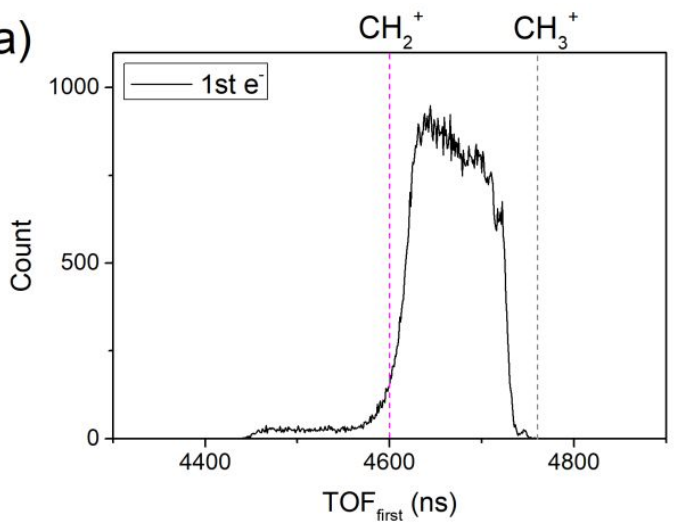

(c)

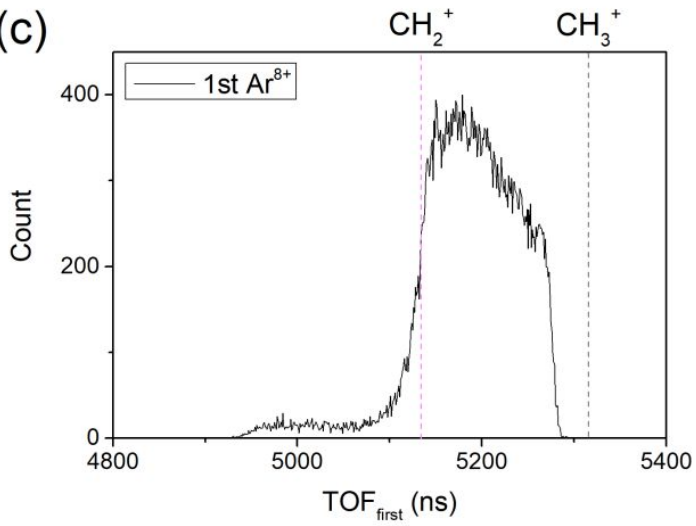

(b)

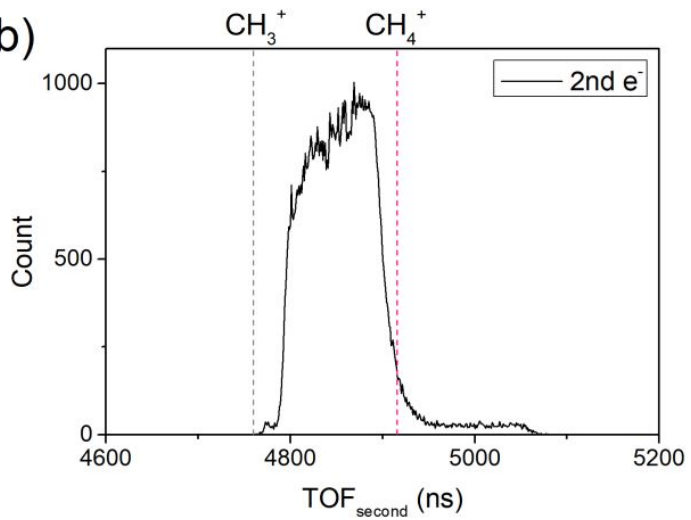

(d)

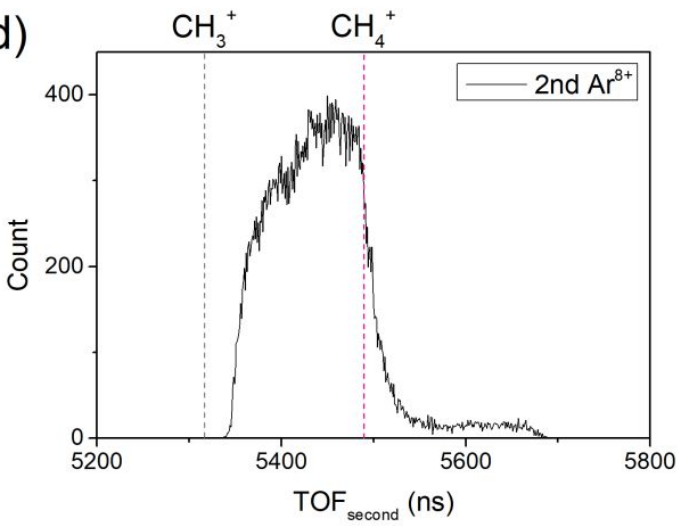

Figure $\mathbf{S 2}$ The 1D TOF spectra of overlapped two-body fragmentation channels $\mathrm{CH}_{3}{ }^{+}+\mathrm{CH}_{3}{ }^{+}$and $\mathrm{CH}_{2}{ }^{+}+\mathrm{CH}_{4}{ }^{+}$. (a) and (b) are the TOFs of the first and second hit recoil ions induced by $300-\mathrm{eV}$ electron, respectively. (c) and (d) are the TOFs of the first and second hit recoil ions induced by 3 $\mathrm{keV} / \mathrm{u} \mathrm{Ar}^{8+}$, respectively.

\section{Branching ratio calculations}

To obtain the branching ratios, that is, to reconstruct the true counts of both $\mathrm{CH}_{3}{ }^{+}+$ $\mathrm{CH}_{3}{ }^{+}$and $\mathrm{CH}_{2}{ }^{+}+\mathrm{CH}_{4}{ }^{+}$channels, we draw diagrams showing the correlation between the fragmentation angle $\theta$ ( $\theta$ represents the angle between the fragmentation direction and TOF axis) and KER, as shown in Figure S3. Since the fragmentation of the twobody channels follows the conservation of momentum and isotropic properties (as a 
result of the random orientation of ethane target molecules, and thus $\theta$ obeys to a $\sin \theta$ distribution), the KER and $\theta$ are distributed independently of each other, and the correlation diagram of KER vs $\theta$ should be distributed as a vertical strip. Assuming that the events in Figure 1 all come from the symmetric fragmentation channel, then Figure S3(a) can be drawn. Since in this channel the two identical fragments cannot be distinguished, the events are only distributed in the angular range of $0^{\circ}-90^{\circ}$. The missing data points at $80-90^{\circ}$ are due to the influence of the detector deadtime. The vertical strip results from the channel $\mathrm{CH}_{3}{ }^{+}+\mathrm{CH}_{3}{ }^{+}$, while the tilted strip in the orange oval comes from the other channel $\mathrm{CH}_{2}{ }^{+}+\mathrm{CH}_{4}{ }^{+}$. The same operation, considering all data from the asymmetric channel, is performed as shown in Figure S3(b). In this case, the vertical strip results from the channel $\mathrm{CH}_{2}{ }^{+}+\mathrm{CH}_{4}{ }^{+}$, while the tilted strip in the orange oval comes from the other channel $\mathrm{CH}_{3}{ }^{+}+\mathrm{CH}_{3}{ }^{+}$.
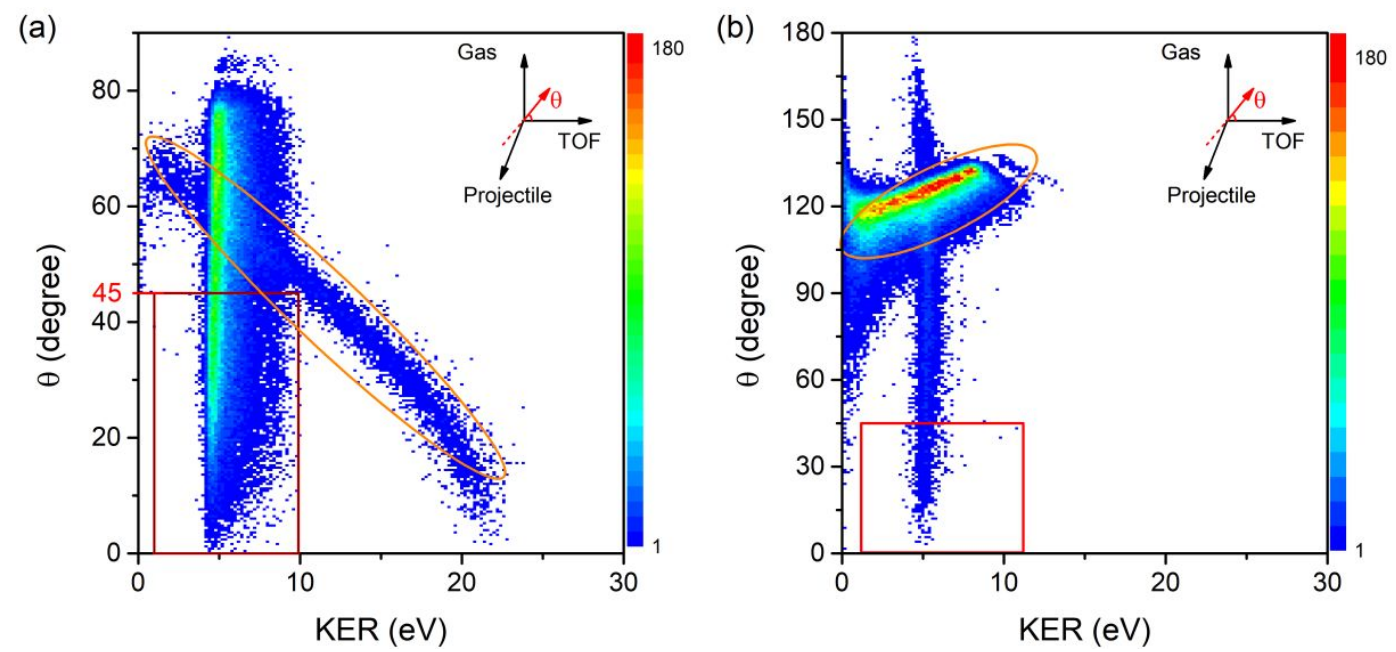

Figure S3 (a) The density plot of KER versus $\theta$. The plot is obtained assuming that all events corresponding to the data in Figure 1 results from the two-body fragmentation channel $\mathrm{CH}_{3}{ }^{+}+\mathrm{CH}_{3}{ }^{+}$. The coordinate chart inserted in the upper right corner is the definition of coordinates adopted in the laboratory, and the $\theta$ represent the angle between the fragmentation direction (red arrow) and TOF axis. (b) The density plot of KER versus $\theta$. The plot is obtained assuming that all events corresponding to the data in Figure 1 results from the two-body fragmentation channel $\mathrm{CH}_{2}{ }^{+}+\mathrm{CH}_{4}{ }^{+}$. The events in orange ovals correspond to false events, which are from $\mathrm{CH}_{2}{ }^{+}+\mathrm{CH}_{4}{ }^{+}$channel in Figure S3(a) and $\mathrm{CH}_{3}{ }^{+}+\mathrm{CH}_{3}{ }^{+}$channel in Figure S3(b), respectively. The branching ratios of $\mathrm{CH}_{3}{ }^{+}$ $+\mathrm{CH}_{3}{ }^{+}$and $\mathrm{CH}_{2}{ }^{+}+\mathrm{CH}_{4}{ }^{+}$channels are calculated from the data within red rectangles.

According to the isotropic feature of a two-body fragmentation channel, we choose 
events with $\theta$ ranging from 0 to $45^{\circ}$ [within red rectangles in Figures S3(a) and (b)] to eliminate the influence of the detector deadtime and the channel overlap in the correlation maps, when calculating the branching ratios of $\mathrm{CH}_{3}{ }^{+}+\mathrm{CH}_{3}{ }^{+}$and $\mathrm{CH}_{2}{ }^{+}+$ $\mathrm{CH}_{4}{ }^{+}$channels. For the symmetrical channel, due to the indistinguishability of recoil fragment ions, half of the event count is considered as the true count. Finally, we can obtain the branching ratios of $\mathrm{CH}_{3}{ }^{+}+\mathrm{CH}_{3}{ }^{+}$and $\mathrm{CH}_{2}{ }^{+}+\mathrm{CH}_{4}{ }^{+}$channels as $\sim 94 \%$ and $\sim 6 \%$, respectively. The branching ratio has a margin of error of $\sim 15 \%$, which was mainly contributed by the statistical errors and the detection efficiency.

In addition, the similar operations have been done for events within black and red rectangles in Figure 1 as shown in Figure S4(a) and (b), respectively. We can draw a conclusion that the data within the black rectangle in Figure 1 is almost entirely (more than $\sim 97 \%$ ) contributed by the coincidence events $\mathrm{CH}_{3}{ }^{+}+\mathrm{CH}_{3}{ }^{+}$, and the events within the red rectangle are attributed only to the dissociation channel $\mathrm{CH}_{2}{ }^{+}+\mathrm{CH}_{4}{ }^{+}$.
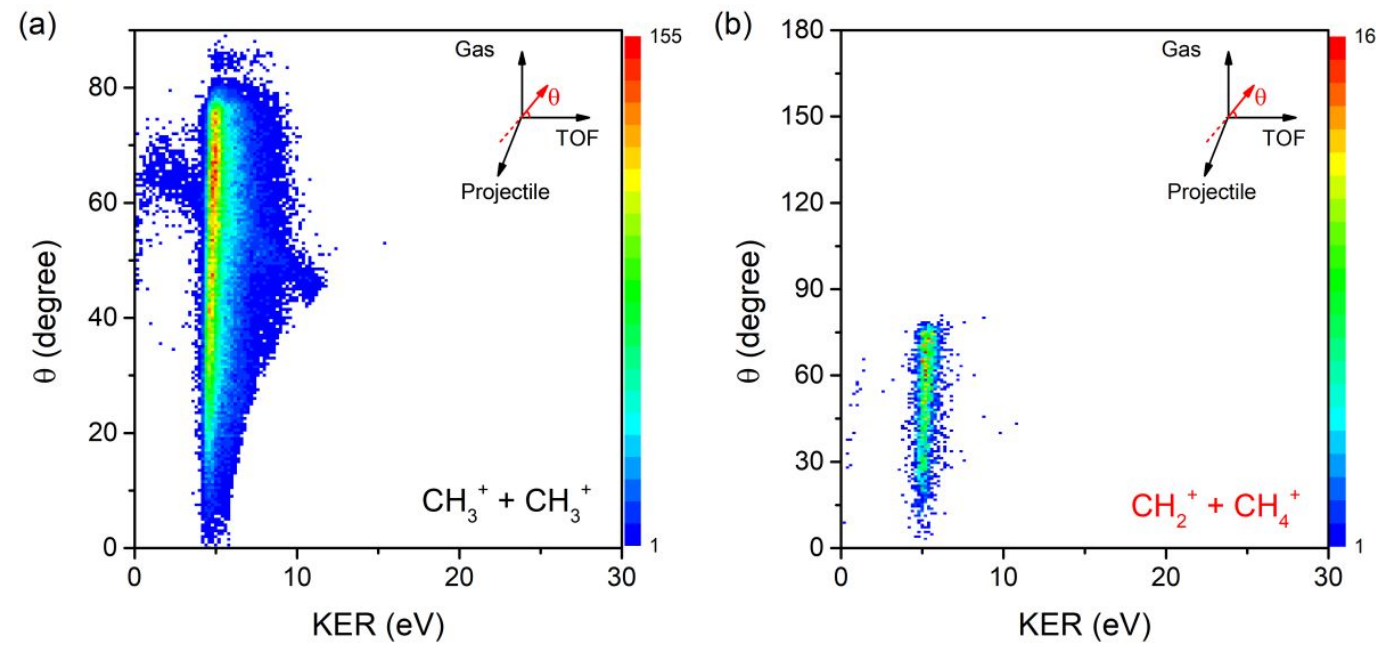

Figure S4 (a) The density plot of KER versus $\theta$ for the data within the black rectangle in Figure 1.

(b) The density plot of KER versus $\theta$ for the data within the red rectangle in Figure 1. 